Concetta Anna Dodaro\#, Armando Calogero*\#, Vincenzo Tammaro, Tommaso Pellegrino, Ruggero Lionetti, Silvia Campanile, Marsela Menkulazi, Massimo Ciccozzi, Anna Maria Iannicelli, Francesco Giallauria, Caterina Sagnelli\#

\title{
Colorectal cancer in the elderly patient: the role of neo-adjuvant therapy
}

https://doi.org/10.1515/med-2019-0068

received March 6, 2019; accepted June 17, 2019

\begin{abstract}
Background: Neoadjuvant chemoradiotherapy has a significant role in downstaging cancer. It improves the local control of the disease and can make conservative resection of rectal cancer possible.
\end{abstract}

Methods: We enrolled 114 patients with subperitoneal rectal cancer who underwent neoadjuvant chemoradiotherapy and radical excision with total mesorectal excision (TME). The primary endpoint was oncological outcomes and the secondary endpoint was surgical outcomes.We evaluate the experience of a multidisciplinary team and the role of neoadjuvant chemoradiotherapy in integrated treatment of cancer of the subperitoneal rectum.

Results: Surgical procedures performed were abdominal perineal resection in 4 cases $(3.5 \%)$, anterior resection in 89 cases (78\%), Hartmann's procedure in 5 cases (4.4\%), and ultralow resection with coloanal anastomosis and diverting stoma in 16 patients (14\%).

\footnotetext{
*Corresponding author: Armando Calogero,Department of Advanced Biomedical Sciences, University of Naples Federico II, Naples, Italy, E-mail: armando.calogero2@unina.it Concetta Anna Dodaro, Vincenzo Tammaro, Tommaso Pellegrino, Silvia Campanile, Marsela Menkulazi, Department of Advanced Biomedical Sciences, University of Naples Federico II, Naples, Italy Ruggero Lionetti, Department of Public Health, University of Naples Federico II, Naples, Italy

Massimo Ciccozzi, Medical Statistics and Molecular Epidemiology Unit, Campus Bio-Medico University, Rome, Italy

Anna Maria Iannicelli, Francesco Giallauria, Department of Translational Medical Sciences, University of Naples “Federico II", Naples, Italy

Caterina Sagnelli, Department of Mental Health and Public Medicine, University of Campania Luigi Vanvitelli, Naples, Italy

\#equally contributed to this work, designed the study and wrote the manuscript.
}

Local recurrence occurred in 6 patients (5.2\%), the overall survival was $71.9 \%$ at 5 years and disease-free survival was about $60 \%$.

Conclusions: The effect of pathological downstaging amounted to $58.8 \%$, including cPR. The pathologic complete remission occurred in $8.8 \%$ of cases.

The outcomes of neoadjuvant therapy can be achieved when this treatment is associated with correct surgical technique with TME and the prognosis is defined by an anatomopathological examination performed according to Quirke's protocol.

Keywords: Neoadjuvant chemoradiotherapy; Colorectal tumors; Rectal cancer

\section{Introduction}

Over the past two decades, outcomes in the management of rectal cancer have improved thanks to a multidisciplinary approach that involves the surgeon, pathologist, radiotherapist and oncologist. There have been continuous efforts to improve local control and long-term survival in patients with rectal cancer, and the introduction of neoadjuvant therapy protocols in the treatment of this tumor has permitted the downstaging of the neoplasm in many cases.

Neoadjuvant therapy has also led to the increase of number of conservative interventions, as well as improving the prognosis by reducing the incidence of recurrence and increasing survival [1-6]. On the other hand surgery alone has a high rate of complications (10-65\%) and local recurrence (10-29\%) [7].

Recently, several studies have generated considerable interest in the neoadjuvant treatment, which consists of the administration of chemotherapy combined with radiation therapy (RT) before surgery, with the aim of 
enhancing the local action of radiation and to sterilize the nodal micrometastases, thus increasing the possibility of achieving radical oncological surgery and sphincter-saving operations [8-13].

In patients undergoing pre-operative RT, there is an objective response rate of $65-70 \%$ and $5-10 \%$ of complete responses; the pre-operative RT significantly reduces the rate of local recurrence and prolongs survival [12,14].

The preoperative chemoradiotherapy has a lower toxicity because it acts at an earlier stage in respect to the development of metastatic cells [15-17].

In this study, we assessed 114 patients with subperitoneal rectal cancer whounderwent preoperative neoadjuvant chemoradiotherapy and total mesorectal excision (TME), from January 2004 to December 2013 at Department of Advanced Biomedical Sciences, University of Naples Federico II, Naples, Italy. We evaluated through the experience of a multidisciplinary team in an observational real-life retrospective study, and the role of neoadjuvant chemoradiotherapy in the integrated treatment of cancer of the lower rectum in the light of oncology and clinical factors outcomes.

\section{Methods}

From January 2004 to December 2013 at our center were admitted 376 patients (206 male and 170 females) with colorectal cancer in an observational real-life retrospective study. Of these 376, 189 patients had adenocarcinomas of the colon and 187 patients had adenocarcinomas of the rectum. Among them, 150 patients showed a cancer of the rectum localized in the subperitoneal space $(<12 \mathrm{~cm}$ from the anal verge). The 150 patients with subperitoneal rectal cancer were evaluated (82 male and 68 female, average age 55 years, range 26-75 years) and were submitted to the following examinations: routine blood chemistry, research of tumor markers, colonscopy, endorectal ultrasound, MRI, CT/PET Total body.

Ethical approval: The research related to human use has been complied with all the relevant national regulations, institutional policies and in accordance the tenets of the Helsinki Declaration, and has been approved by the authors' institutional review board or equivalent committee. At the first observation, each patient gave signed informed consent to undergot the surgical procedure and be a part of the clinical research.

The study protocol had the following exclusion criteria: beyond the intraperitoneal tumor site $(>12 \mathrm{~cm}$ from the anal verge) (11 cases); histology different from ade- nocarcinoma ( 3 squamous cancer of the anus; 2 lymphomas); synchronous primitive multiple cancers (MPM) (6 cases); presence of metastases (8 cases); and T1, T4 and T2No (6 cases).

Of the 150 patients, 114 patients were enrolled and treated with neoadjuvant therapy.

The synthesis before and after neoadjuvant treatment of 114 patients is reported in Table 1.

The treatment given to patients is constituted by one of concomitant chemotherapy scheme $[18,19]$.

\subsection{External radiation therapy (RT)}

Radiation treatment was given using a linear accelerator (6-25 MV) and isocentric technique of 3 fields (one posterior and 2 lateral) with a total dose of $50.4 \mathrm{~Gy}$ ( 25 daily fractions of $1.8 \mathrm{~Gy}$ to the pelvis plus 3 additional daily fractions of $1.8 \mathrm{~Gy}$ to the tumor). The entire treatment had a duration of 28 days.

\subsection{Chemotherapy (CHT)}

Continuous infusion i.v. with elastomeric pump of 5-fluorouracil at dose of $225 \mathrm{mg} / \mathrm{m} 2$ for the duration of the radia-

Table 1: Demographic, clinical and surgical procedures in 114 patients

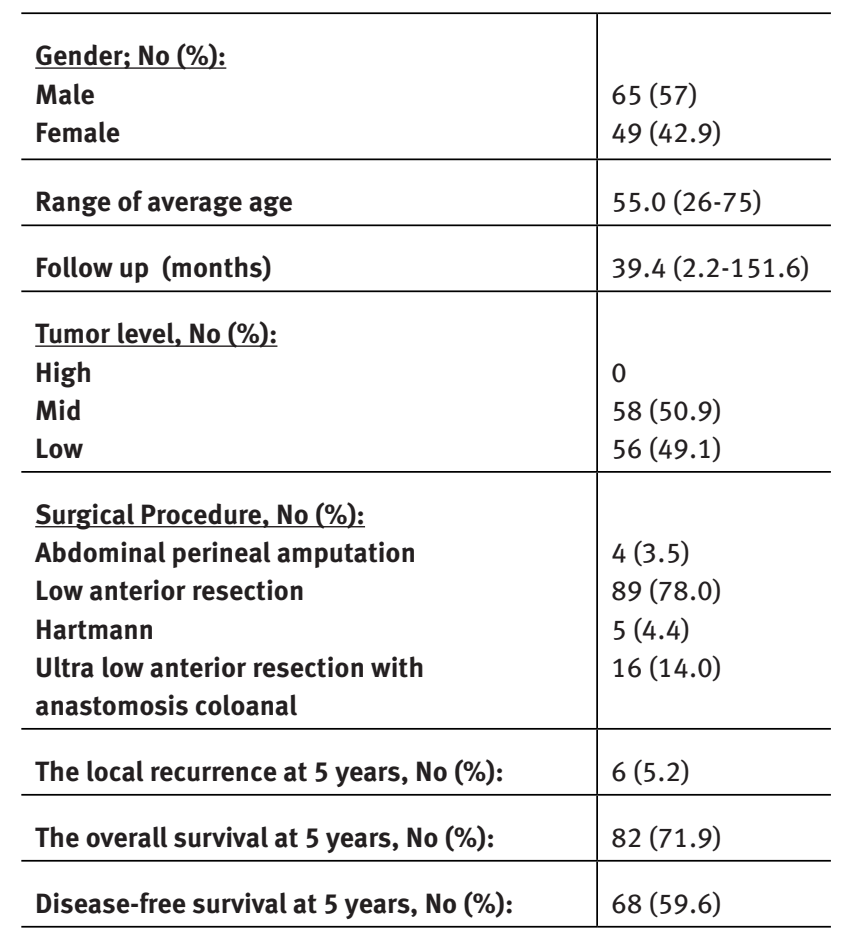


tion treatment. Two boluses i.v. week of oxaliplatin at dose of $60 \mathrm{mg} / \mathrm{m} 2$.

The patients were re-admitted at our center 4-6 weeks after the end of neoadjuvant therapy in order to perform restaging and undergo surgery between 6 th and 8th week after the end of preoperative chemoradiotherapy.

For all patients at the time of initial staging, we assessed whether or not to perform a conservative surgery of the sphincter function, essentially based on the distance of the tumor from the anal verge.

The follow-up after surgery was standard with sixmonthly checks for at least 5 years.

All patients were followed up by the surgeon, specialist oncologist, and radiotherapist, and were subjected to careful clinical evaluation, blood chemistry with evaluation of the levels of CEA, and chest X-ray.

\section{Results}

We enrolled 114 consecutive patients, 65 were male and 49 female with a mean age 55 years and range 26-75 years. All cases had histologically determined adenocarcinoma of the rectum. They were considered only when the rectal cancer localization was $\leq 12 \mathrm{~cm}$ from the anal verge.

All patients underwent clinical-laboratory weekly monitoring during the course of concomitant chemotherapy, which showed no toxic effects, with a performance status of ECOG grade 0 (patient physically active can perform normal activities without restriction). Only two patients presented a moderate neutropenia. All 114 patients completed the set cycle.

The surgical procedures performed were: abdominal perineal resection in 4 cases $(3.5 \%)$, anterior resection in 89 cases $(78 \%)$, Hartmann's procedure in 5 cases $(4.4 \%)$, and ultralow resection with coloanal anastomosis and diverting stoma in 16 patients (14\%) (Table 1).

The mean number of lymph nodes examined was 12; the hospital stay was 9-25 days. Seventeen patients had postoperative complications: postoperative wound dehiscence in $3(2.6 \%)$ patients, anastomotic leak in $9(7.9 \%)$ patients, major cardiovascular complications in 5 (4.4\%) patients (Table 2).

In all 114 patients the resection margins assessed with pathologic examination of the surgical specimen had no residual tumor cells.

During follow-up, we detected two cases of local recurrence (1.8\%) at 6 and 15 months respectively. Both patients had lymph node recurrence after surgery, and one of them developed liver metastases. The local recurrence occurred in 6 patients (5.2\%) at 5 years, the overall survival at 5 years was observed in $82(71.9 \%)$ patients and disease-free survival at 5 years in 68 patients (59.6\%).

\section{Conclusion}

In recent years, the use of neoadjuvant chemoradiotherapy has increased in the perioperative treatment of rectal cancer [20-22]. Several studies show that the combination of a "short course" of neoadjuvant radiotherapy and total mesorectal excision results in a better local control than only TME [21]. In addition, the neoadjuvant chemoradiotherapy has been introduced in the treatment of locally advanced rectal cancer to improve the preservation of the anal sphincter [14,22-24]. In fact, when the rectal cancer is locally advanced at diagnosis, recurrence after surgical resection occurs in a range of $50 \%$ to $70 \%$ of cases and is characterized by the early onset of distant metastases [25]. The neoadjuvant chemoradiotherapy is part of a safe and efficient multimodal treatment of rectal cancer. It shows significant benefits in terms of reducing the tumor volume and less compromise to the neoplastic lymph node, thus confirming the concept of down staging.

In this study, the effect of pathological down staging amounted to $58.8 \%$, including cPR. The pathologic complete remission occurred in $8.8 \%$ of cases. Patients who had a complete pathological remission have had good outcomes in terms of OS and DFS $(71,3 \%$ and $60 \%$, respectively).

In locally advanced rectal carcinoma, this combined treatment approach aims to improve resectability, local control and overall survival. It does so by reducing the tumor volume thus favoring surgical resection and longterm oncological results [26-28].

In our experience we found a clinical and instrumental down staging in 59 patients (51.75\%). This finding is in agreement with literature, the neoadjuvant treatment for carcinoma of the rectum can cause a down staging which permits an increase of conservation of the sphincter function [21-24,29-31].

Table 2: Postoperative complication in 17 patients.

Postoperative complications, $\mathrm{n}$ (\%):

\begin{tabular}{ll}
\hline Wound dehiscence & $3(2.6 \%)$ \\
Anastomotic leak & $9(7.9 \%)$ \\
Major cardiovascular complications & $5(4.4 \%)$ \\
\hline
\end{tabular}


Many trials also show that the neoadjuvant chemoradiotherapy appears associated with a decrease in the rate of local recurrence and to an increase in the overall survival rate [18]. However, these data are not accepted univocally as some authors argue that the neoadjuvant chemoradiotherapy, though it determines a down staging and, sometimes, a complete "eradication" of the tumor histology, is complicated by high toxicity and a high rate of anastomotic leak [32].

In agreement with this finding, in patients subjected to ultralow colo-rectal anastomosis we always made a temporary ileostomy [33].

Some authors emphasize the role of lymph nodes involvement detected on the histological sample. In fact in patients treated with preoperative chemoradiotherapy the presence of positive lymph nodes in the histological sample would be associated with a worse prognosis $[34,35]$. Hence the importance of intraoperative lymph node metastases [36].

In our study, the preoperative staging of rectal cancer was performed with the digital rectal examination, transrectal ultrasound, colonoscopy and magnetic resonance imaging (MRI).

Nowadays EUS is considered very accurate for $\mathrm{T}$ staging, because it can precisely define the degree of tumor penetration into the various layers of the rectal wall [37-41]. MRI is the best method to define distance of the mesorectal fascia from the lateral margin of the tumor and so to determine which patients can benefit from neoadjuvant therapy. Moreover, its accuracy is greater than ultrasonography in identifying the tumors [35, 42-47].

The pathological complete response occurred in 10 patients $(8.8 \%)$, like observed by Grann et al. in $9 \%$ of cases [9]. However, if the cancer has been shown to have a pathological downstaging, the latter can provide related benefits to the resection of the tumor and the improvement of the oncological results [26-27].

The final objective of preoperative chemoradiotherapy was the pathological complete remission. The best outcomes of neoadjuvant therapy can be achieved when this treatment is associated with a correct surgical technique with TME and the prognosis is defined by an anatomopathological examination performed according to Quirke's protocol.

Conflict of interest: The authors have no conflicts of interest (political, personal, religious, ideological, academic, intellectual, commercial or any other) to declare in relation to this manuscript.
Competing Interests: The authors have declared that no competing interests exist.

\section{References}

[1] De Felice F, Daniela Musio D, Izzo L, Tombolini V. Neoadjuvant chemoradiotherapy for locally advanced rectal cancer: The debate continues. World J Gastrointest Oncol. 2014 Dec 15;6(12):438-40. doi: 10.4251/wjgo.v6.i12.438

[2] Schrag D. Evolving role of neoadjuvant therapy in rectal cancer. Curr Treat Options Oncol. 2013 Sep;14(3):350-64. doi: 10.1007/s11864-013-242-248

[3] Boland PM, Fakin M. The emerging role of neoadjuvant chemotherapy for rectal cancer. J Gastrointest Oncol. 2014 Oct;5(5):362-73. doi: 10.3978/j.issn. 2078-6891.2014.060

[4] Arnold D, Schmoll HJ. (Neo-)adjuvant treatments in colorectal cancer. Ann Oncol. 2005. 16 Suppl 2: ii133-40

[5] Kotake K, Koyama Y, Shida S, Tajima Y, Ishikawa H, Kanazawa $\mathrm{K}$, et al. Neo-adjuvant chemotherapy with carmofur for colorectal cancer--a multi-institutional randomized controlled study. Gan To Kagaku Ryoho. 2002. 29(11): 1917-1924

[6] Kotake K, Koyama Y, Konishi F, Kanazawa K, Namba M, Shida $S$, et al. Neo-adjuvant chemotherapy with tegafur suppository for rectal cancer--evaluation of the antitumor effects, tissue levels of 5-FU and inhibition of thymidylate synthase. Tochigi Colorectal Cancer Study Group. Gan To Kagaku Ryoho. 1992. 19(11): 1837-1842

[7] Santangelo ML, Grifasi C, Criscitiello C, Giuliano M, Calogero A, Dodaro C, et al. Bowel obstruction and peritoneal carcinomatosis in the elderly. A systematic review. Aging Clin Exp Res. 2017 Feb;29(Suppl 1):73-78. doi: 10.1007/s40520-0160656-9

[8] Carlomagno N, Calogero A, Saracco M, Santangelo M, Dodaro C, Renda A. Simultaneous quadruple carcinoma of colon Case report and literature review. Ann Ital Chir. 2014;85:495-500

[9] Grann A, Minsky BD, Cohen A M, Saltz L, Guillem JG, Paty PB, et al. Preliminary results of preoperative 5-fluorouracil, low-d ose leucovorin, and concurrent radiation therapy for clinically resectable T3 rectal cancer. Diseases of the Colon \& Rectum. 1997 May;40(5):515-522

[10] Martelluci J, Alemanno G, Castiglione F, Bergamini C, Valeri A. Role of KRAS mutation as predictor of pathologic response after neoadjuvant chemoradiation therapy for rectal cancer. Updates Surg. 2015 Mar;67(1):47-53. doi: 10.1007/s13304015-0281-8

[11] Grifasi C, Calogero A, Esposito A, Dodaro C. Perioperative care of elderly outpatients. A review. Ann Ital Chir 2015 Mar-Apr;86(2):100-105

[12] Read TE, McNevin MS, Gross EKM, Whiteford HM, Lewis JL, Ratkin G, et al. Neoadjuvant therapy for adenocarcinoma of the Rectum: Tumor response and acute toxicity. Disease Colon \& Rectum 2001 Apr; 44 (4): 513-522

[13] Carlomagno N, Santangelo M, Amato B, Calogero A, Saracco M, Cremone C, et al. Total colectomy for cancer: analysis of factors linked to patients' age. Int J Surg. 2014;12 Suppl 2:S135-9. doi: 10.1016/j.ijsu.2014.08.363 
[14] Wolmark N, Fisher B. An analysis of survival and treatment failure following abdominoperineal and sphincter-saving resection in Dukes' $B$ and $C$ rectal carcinoma. A report of the NSABP clinical trials. National Surgical Adjuvant Breast and Bowel Project. Ann Surg. 1986;204:480-489

[15] Carlomagno N, Santangelo M, Mastromarino R, Calogero A, Dodaro C, Renda A. Rare multiple primary malignancies among surgical patients-a single surgical unit experience. Ecancermedicalscience 2014 Jun 18;8:438. doi: 10.3332/ ecancer.2014.438

[16] Sclafani F, Cunningham D. Neoadjuvant chemotherapy without radiotherapy for locally advanced rectal cancer. Future Oncol. 2014 Nov;10(14):2243-57. doi: 10.2217/ fon.14.127

[17] Nastro P, Sodo M, Dodaro C, Gargiulo S, Acampa W, Bracale $\mathrm{U}$, Renda A. Intraoperative radiochromoguided mapping of sentinel lymph node in colon cancer. Tumori 2002 Jul-Aug;88(4):352-353

[18] Das P, Skibber JM, Rodriguez-Bigas MA, Feig BW, Chang GJ, Wolff RA, et al. Predictors of tumor response and downstaging in patients who receive preoperative chemoradiation for rectal cancer. Cancer 2007 May 1;109(9):1750-1755

[19] Janjan NA, Khoo VS, Abbruzzese J, Pazdur R, Dubrow $\mathrm{R}$, Cleary KR, et al. Tumor downstaging and sphincter preservation with preoperative chemoradiation in locally advanced rectal cancer: the M. D. Anderson Cancer Center experience. Int J Radiat Oncol Biol Phys 1999 Jul 15;44(5):1027-1038

[20] Folkesson J, Birgisson H, Pahlman L, Cedermark B, Glimelius $B$, Gunnarsson U. Swedish Rectal Cancer Trial: long lasting benefits from radiotherapy on survival and local recurrence rate. Clin Oncol. 2005; 23:5644-5650

[21] Tjandra JJ, Reading DM, Gunn IF, Green MD, McLaughlin SJ, et al. Phase II clinical trial of preoperative combined chemoradiation for T3 and T4 resectable rectal cancer: prelminary results. Disease Colon \& Rectum. 2000 Aug; 44(8): 1113-1122

[22] Rega D, Pecori B, Scala D, Avallone A, Pace U, Petrillo A, et al. Evaluation of Tumor Response after Short-Course Radiotherapy and Delayed Surgery for Rectal Cancer. Plos One 2016 Aug 22;11(8):e0160732. doi: 10.1371/journal. pone. 0160732

[23] Cuccurullo D, Pirozzi F, Sciuto A, Bracale U, La Barbera C, Galante F, et al. Relaparoscopy for management of postoperative complications following colorectal surgery: ten years experience in a single center. Surg Endosc. 2015 Jul;29(7):1795-803. doi: 10.1007/s00464-014-3862-6

[24] Glynne-Jones R, Wallace M, Livingstone JIL, Meyrick-Thomas J. Complete clincal response Aeer preoperative Chemoradiation in Rectal Cancer: Is a "Wait and See" Policy Justified? Disease Colon \& Rectum.2008 Jan, 51(1): pp 10-12

[25] Liu KT, Wan JF, Zhu J, Li GC, Sun WJ, Shen LJ, et al. Role of pelvic radiotherapy for locally advanced rectal cancer and synchronous unresectable distant metastases. Cancer Radiother 2016 Dec;20(8):805-810. doi: 10.1016/j. canrad.2016.06.009

[26] Caccavale S, Vitiello P, Franco R, Panarese I, Ronchi A, Sica A, et al. Dermoscopic characterization of folliculotropic mycosis fungoides selectively localized on trunk and limbs.Int J Dermatol. 2019 May 28. doi: 10.1111/ijd.14490
[27] Gambacorta MA, Valentini V, Coco C, Manno A, Doglietto $G B$, Ratto $C$, et al. Sphincter Preservation In four consecutive phase II studies of preoperative chemoradiation: Analysis of 247 T3 rectal cancer patients.Tumori. 2007 Mar-Apr; 93(2):160-169

[28] Grillo-Ruggeri F, Mantello G, Cardinali M, Fabbietti L, Fenu F, Montisci M et al. Downstaging after two different preoperative chemoradiation schedules in rectal cancer. Tumori. 2003 Mar- Apr; 89(2): 164-167

[29] Peluso G, Incollingo P, Calogero A, Tammaro V, Rupealta $\mathrm{N}$, Chiacchio G, et al. Current tissue molcular markers in colorectal cancer: A literature review Biomed Res Int. 2017;2017:2605628. doi: 10.1155/2017/2605628

[30] Nardelli C, laffaldano L, Pilone V, Labruna G, Ferrigno M, Carlomagno N, et al. Changes in the MicroRNA Profile Observed in the Subcutaneous Adipose Tissue of Obese Patients after Laparoscopic Adjustable Gastric Banding. J Obes. 2017;2017:6754734. doi: 10.1155/2017/6754734

[31] Dodaro CA, Grifasi C, Lo Conte D, Romagnuolo R. Advantages and disadvantages of day surgery in a department of general surgery. Ann Ital Chir. 2013 Jul-Aug;84(4):441-4. PMID: 23103718

[32] Georgiou P, Tan E, Gouvas N, Antoniou A, Brown G, Nicholls RJ, et al. Extended lymphadenectomy versus conventional surgery for rectal cancer: a meta-analysis. Lancet Oncol. 2009;10:1053-1062

[33] Bujko K, Nowacki MP, Nasierowska-Guttmejer A, Michalski W, Bebenek M, Kryj M. Long-term results of a randomized trial comparing preoperative short-course radiotherapy with preoperative conventionally fractionated chemoradiation for rectal cancer. Br J Surg. 2006;93:1215-1223

[34] Gualdi GF, Casciani E, Guadalaxara A, D’Orta C, Polettini E, Pappalardo G. Local staging of rectal cancer with transrectal ultrasound and endorectal magnetic resonance imaging: comparison with histologic findings. Disease Colon \& Rectum. 2000 Mar;43(3): 338-453

[35] Panzironi G, Guerrieri D, De Cristofaro F, Bangrazi C, Di Paola $C$, Jafari L, et al. Endorectal ultrasonography performance in staging rectal cancer before and aeer neoadjuvant chemoradiotherapy. Ann Ital Chir. 2014;85:569-575

[36] Kim HJ, Wong WD. Role of Endorectal Ultrasound in the Conservative Management of Rectal Cancer. Semin Surg Oncol. 2000 Dec; 19(4): 336-349

[37] Garcia-Aguilar J, Pollack J, Lee SH, Hernandez de Anda E, Mellgren A, Wong WD, et al. Accuracy of endorectal ultrasonography in preoperative staging of rectal tumors. Disease Colon \& Rectum. 2002;45:10-15

[38] Marusch F, Koch A, Schmidt U, Zippel R, Kuhn R, Wolff S, et al. Routine use of transrectal ultrasound in rectal carcinoma: results of a prospective multicenter study. Endoscopy. 2002;34:385 390

[39] Kim NK, Kim MJ, Yun SH, Sohn SK, Min JS. Comparative study of transrectal ultrasonography, pelvic computerized tomography, and magnetic resonance imaging in preoperative staging of rectal cancer. - Disease Colon \& Rectum. 1999 Jun; 42(6):770-577

[40] Calogero A, Sagnelli E, Creta M, Angeletti S, Peluso G, Incollingo $\mathrm{P}$, et al. Eradication of HCV infection with the Direct-Acting Antiviral Therapy in renal allograft recipients BioMed Research International 2019,4674560, doi: 
10.1155/2019/4674560. "Erratum to "Eradication of HCV Infection with the Direct-Acting Antiviral Therapy in Renal Allograft Recipients", BioMed Research International, vol. 2019, Article ID 8797329, 1 pages, 2019. https://doi. org/10.1155/2019/8797329

[41] Mercury Study Group Diagnostic accuracy of preoperative magnetic resonance imaging in predic;ng curative resection of rectal cancer: prospective observational study. Br Med J. 2006; Oct 14;333(7572):779

[42] Fuchsjager MH, Maier AG, Schima W, Zebedin E, Herbst F, Mittlböck M, et al. Comparison of transrectal sonography and double-contrast MR imaging when staging rectal cancer. AJR Am J Roentgenol. 2003 Aug;181(2):421-427

[43] Wallengren NO, Holtas S, Andren-Sandberg A, Jonsson E, Kristoffersson DT, McGill S. Rectal carcinoma: double-contrast
MR imaging for preoperative staging. Radiology $2000 \mathrm{Apr}$; 215(1): 108-114

[44] Dodaro C, Grifasi C, Florio J, Santangelo M, Duraturo F, De Rosa M, et al. The role of mutation analysis of the APC gene in the management of FAP patients. A controversial issue. Ann Ital Chir. 2016;87:321-325

[45] Carlomagno C, Farella A, Bucci L, Pesce G, Pepe S, Cannella L, et al. Neo-adjuvant treatment of rectal cancer with capecitabine and oxaliplatin in combination with radiotherapy: a phase II study. Ann Oncol. 2009;20:906-912

[46] Ren JH, Guo FJ, Dai WD, Han XJ, Ma N. Study of endorectal ultrasonography in the staging of rectal cancer. Chin Med J (Engl). 2012 Oct;125(20):3740-3743

[47] Santangelo ML, Criscitiello C, Garcia-Aguilar j. Imaging in rectal cancer: magneticc resonance imaging versus endorectal ultrasonography. Surg Oncol Clin N Am. 2014 Jan;23(1):59-77. doi: 10.1016/j.soc.2013.09.011. 